J. DIFFERENTIAL GEOMETRY

45 (1997) 74-93

\title{
ON THE TOPOLOGICAL ENTROPY OF GEODESIC FLOWS
}

\author{
RICARDO MAÑÉ
}

\section{Introduction}

Let $M^{n}$ be a closed connected $C^{\infty}$ manifold and let $S M$ be its unit tangent bundle, defined as usual as $S M=\{\theta=(x, v): x \in M, v \in$ $\left.T_{x} M,\|v\|=1\right\}$. The geodesic flow $\varphi_{t}: S M \rightarrow S M$ is defined by $\varphi_{t}(x, v)=(\gamma(t), \dot{\gamma}(t))$, where $\gamma: \mathbf{R} \rightarrow M$ is the geodesic with initial conditions $\gamma(0)=x$ and $\dot{\gamma}(0)=v$.

Given $x$ and $y$ in $M$, define $n_{T}(x, y)$ as the number of geodesics of length $\leq T$ (parametrized by arc length) joining $x$ and $y$. A standard application of Sard's Theorem to the exponential maps of $M$ shows that $n_{T}(x, y)$ is finite and locally constant on an open full measure subset of $M \times M$.

Our aim is to relate the exponential growth rate of $n_{T}(x, y)$, as a function of $T$, with the topological entropy of the geodesic flow $h_{t o p}(\varphi)$. In that direction, among other results, we shall prove that

$$
h_{\text {top }}(\varphi)=\lim _{T \rightarrow+\infty} \frac{1}{T} \log \int_{M \times M} n_{T}(x, y) d x d y .
$$

While proving this result, we shall also prove that Przytycki's upper estimate for the topological entropy of general $C^{2}$ flows [8], is always an equality for $C^{\infty}$ geodesic flows. Since Przytycki's inequality will be a key tool in our proofs we begin by recalling its statement. Given a

Received November 21, 1994, and, in revised form July 11, 1995.

The author died while the paper was being refereed. The suggested revisions were made by Keith Burns, Gabriel Paternain and Miguel Paternain. 
linear map $L: E \rightarrow F$ between finite dimensional Hilbert spaces, we define its expansion ex $(L)$ by

$$
\operatorname{ex}(L)=\max _{S}\left|\operatorname{det}\left(\left.L\right|_{S}\right)\right|
$$

where the maximum is taken over all subspaces $S \subset E$. Przytycki's inequality states that for a $C^{2}$ flow $\psi_{t}: N \rightarrow N$ on a closed manifold $N$,

$$
h_{\text {top }}(\psi) \leq \liminf _{t \rightarrow \infty} \frac{1}{t} \log \int_{N} e x\left(d_{x} \psi_{t}\right) d x .
$$

The main result of this paper is Theorem 1.1 below. Half of its proof relies on a combination of Yomdin's theorem [9] and a formula due to Berger and Bott [2] that, although not difficult to prove, provides the link between the numbers $n_{T}(x, y)$ and the dynamics of the geodesic flow. We took this combination from [6], but for our purposes an improved version of Yomdin's theorem will be needed. The other half relies on Przytycki's inequality, Berger and Bott's formula, and a careful change of variables, where the novelty of this work resides.

\section{Theorem 1.1.}

$$
\begin{aligned}
h_{\text {top }}(\varphi) & =\lim _{T \rightarrow+\infty} \frac{1}{T} \log \int_{M \times M} n_{T}(x, y) d x d y \\
& =\lim _{T \rightarrow+\infty} \frac{1}{T} \log \int_{S M} e x\left(d_{\theta} \varphi_{T}\right) d \theta .
\end{aligned}
$$

When the manifold has no conjugate points, it is easy to check, using the fact that the exponential map $\exp _{x}: T_{x} M \rightarrow M$ is a covering map admitting a fundamental domain of diameter $\leq \operatorname{diam}(M) \stackrel{\text { def }}{=} c$, that for any $x_{1}, y_{1}, x_{2}, y_{2} \in M$,

$$
n_{T}\left(x_{1}, y_{1}\right) \leq n_{T+2 c}\left(x_{2}, y_{2}\right)
$$

This property and Theorem 1.1 imply:

Corollary 1.2. If $M$ has no conjugate points, then

$$
\lim _{T \rightarrow+\infty} \frac{1}{T} \log n_{T}(x, y)=h_{\text {top }}(\psi)
$$

for all $x, y \in M$. 
From this corollary we can recover the results of Freire and Mañé [3] on the equality of the topological entropy of the geodesic flow and the volume growth rate of the manifold when there are no conjugate points. Recall that the volume growth rate $\lambda(M)$ of $M$ is defined by

$$
\lambda(M)=\lim _{r \rightarrow+\infty} \frac{1}{r} \log \operatorname{Vol}\left(B_{r}(x)\right)
$$

where $B_{r}(x)$ denotes the ball of radius $r$ and center $x$ in the universal covering $\tilde{M}$ of $M$, and $\operatorname{Vol}\left(B_{r}(x)\right)$ denotes its volume. Manning proved $[5]$ that this limit exists and is independent of $x$. Moreover he proved that $\lambda(M) \leq h_{\text {top }}(\varphi)$ for every closed Riemannian manifold and $\lambda(M)=$ $h_{t o p}(\varphi)$ when $M$ has sectional curvatures $\leq 0$. In [3], Freire and Mañé extended this result to manifolds without conjugate points through a different technique (see Remark 1.5 below). Here we can obtain it from Corollary 1.2.

Corollary 1.3. If $M$ has no conjugate points, then

$$
\lambda(M)=h_{t o p}(\varphi) .
$$

To deduce Corollary 1.3 from Corollary 1.2, it suffices to show that $\lambda(M) \geq h_{\text {top }}(\varphi)$, because, as we explained above, the inequality $\lambda(M) \leq$ $h_{\text {top }}(\varphi)$ always holds. If $p: \tilde{M} \rightarrow M$ is the covering map and $x \in \tilde{M}$, then the number $n_{T}(p(x), p(x))$ is just the number of points in the set

$$
G_{T} \stackrel{\text { def }}{=}\{z \in M: p(z)=p(x), d(z, x) \leq T\} .
$$

Observe that there exists $r_{0}>0$ such that any two distinct points $z^{\prime}$ and $z^{\prime \prime}$ with $p\left(z^{\prime}\right)=p\left(z^{\prime \prime}\right)$, satisfy $d\left(z^{\prime}, z^{\prime \prime}\right) \geq r_{0}$. Clearly

$$
B_{T+r_{0}}(x) \supset \bigcup_{z \in G_{T}} B_{r_{0}}(z),
$$

and the sets $B_{r_{0} / 2}(z), z \in G_{T}$ are disjoint. Hence if

$$
k \stackrel{\text { def }}{=} \min \left\{\operatorname{Vol}\left(B_{r_{0} / 2}(a)\right): a \in \tilde{M}\right\}
$$

we have

$$
\operatorname{Vol}\left(B_{T+r_{0}}(x)\right) \geq k \# G_{T}=k n_{T}(p(x), p(x)) .
$$


Therefore

$$
\begin{aligned}
\lambda(M) & =\lim _{T \rightarrow+\infty} \frac{1}{T+r_{0}} \log \operatorname{Vol}\left(B_{T+r_{0}}(x)\right) \\
& \geq \lim _{T \rightarrow+\infty} \frac{1}{T+r_{0}} \log k n_{T}(p(x), p(x))=h_{\text {top }}(\varphi),
\end{aligned}
$$

thus completing the proof of the corollary.

In the next statement, $V(\theta)$ will denote the vertical fibre at $\theta=(x, v) \in S M$, defined by

$$
V(\theta)=d_{\theta} \pi^{-1}(\{0\}),
$$

where $\pi$ is the projection map $\pi: S M \rightarrow M$.

Theorem 1.4.

$$
\begin{aligned}
h_{\text {top }}(\varphi) & =\lim _{T \rightarrow+\infty} \frac{1}{T} \log \int_{S M}\left|\operatorname{det}\left(\left.d_{\theta} \varphi_{T}\right|_{V(\theta)}\right)\right| d \theta \\
& =\lim _{T \rightarrow+\infty} \frac{1}{T} \log \int_{M} \operatorname{Vol}\left(\varphi_{T}\left(S_{x} M\right)\right) d x
\end{aligned}
$$

The second equality is trivial because

$$
\operatorname{Vol}\left(\varphi_{T}\left(S_{x} M\right)\right)=\int_{S_{x} M}\left|\operatorname{det}\left(\left.d_{(x, v)} \varphi_{T}\right|_{V(x, v)}\right)\right| d v
$$

Remark 1.5. Manning's proof of Corollary 1.3 for manifolds of non-positive curvature relies on the fact that for such manifolds, any two geodesics $\gamma_{i}: \mathbf{R} \rightarrow \tilde{M}$ in the universal covering satisfy:

$$
d\left(\gamma_{1}(t), \gamma_{2}(t)\right) \leq d\left(\gamma_{1}(0), \gamma_{2}(0)\right)+d\left(\gamma_{1}(T), \gamma_{2}(T)\right)
$$

for all $0 \leq t \leq T$. In [3] the authors observed that for manifolds where this property holds with the right term multiplied by a constant independent of the geodesics, Manning's proof can be applied with insignificant changes, thus providing a much simpler proof of their result if the existence of such a constant could be established for manifolds without conjugate points. However the example of Ballmann, Brin and Burns [1], that appeared four years later, proved that such a constant does not exist. 
Remark 1.6. On the question of the exponential growth rate of $n_{T}(x, y)$, without assumptions like the absence of conjugate points we have little to say; only that (see Appendix) as a corollary of a result of G. Paternain [6], it follows that for every $x \in M$,

$$
\limsup _{T \rightarrow+\infty} \frac{1}{T} \log n_{T}(x, y) \leq h_{t o p}(\varphi)
$$

for a.e. $y \in M$. This naturally poses the following question:

Problem I. Is it true that for a.e. $(x, y) \in M \times M$,

$$
\lim _{T \rightarrow+\infty} \frac{1}{T} \log n_{T}(x, y)=h_{t o p}(\varphi) ?
$$

Since an affirmative answer to this problem may sound too good to be true, a humbler, and more feasible question is the following one:

Problem II. Is it true that (0.1) holds for generic Riemannian metrics when $\operatorname{dim} M=2$ ?

Remark 1.7. Theorem 1.1 above was announced, and its proof sketched, in a preprint of the author circulated in January 93. The proof that we shall use here is essentially shorter than that outlined there.

The proof of Theorem 1.1 relies on three inequalities. Two of them (inequalities $(A)$ and $\left(A^{\prime}\right)$ below) are where the novelty of this paper resides. The other inequality (inequality (B) below) is a variation of an inequality due to G. Paternain [6], which in turn comes from Yomdin's theorem [9]. One way to prove this inequality is to combine G. Paternain's method with a certain uniformity in Yomdin's theorem, visible in Gromov's exposition of this celebrated result in the Bourbaki Seminaire [4]. Such was the method employed in our announcement. Afterwards, G. Paternain and M. Paternain [7] proved that inequality using directly Yomdin's theorem without having to appeal to the uniformity mentioned above.

\section{Proof of Theorem 1.1}

We begin by recalling the basic formalism of geodesic flows, stressing its symplectic properties, which will play a key role in our proofs. 
Given $\theta=(x, v) \in S M$ define

$$
E(\theta)=\left\{w \in T_{x} M:\langle w, v\rangle=0\right\} .
$$

Denote by $\pi: S M \rightarrow M$ the canonical projection and set

$$
\begin{gathered}
N(\theta)=d_{\theta} \pi^{-1}(E(\theta)), \\
V(\theta)=d_{\theta} \pi^{-1}(\{0\}) .
\end{gathered}
$$

If $X$ is the geodesic vector field on $S M$ (i.e., the vector field generated by the geodesic flow), then $T_{\theta} S M$ is the direct sum of $N(\theta)$ and the onedimensional subspace spanned by $X(\theta)$. Moreover, $N$ is $\varphi_{t}$-invariant, i.e.,

$$
d_{\theta} \varphi_{t}(N(\theta))=N\left(\varphi_{t}(\theta)\right),
$$

for all $\theta \in S M$ and $t \in \mathbf{R}$. On each $N_{\theta}$ there exist an inner product $<,>_{\theta}$ and an isometry $J_{\theta}: N(\theta) \rightarrow N(\theta)$ of this inner product, such that for all $\theta$ :

a) $J_{\theta}^{2}=-I$

b) $V(\theta)$ and $J_{\theta} V(\theta)$ are orthogonal;

c) $\left\langle J_{\varphi_{t}(\theta)} d_{\theta} \varphi_{t}(\zeta), d_{\theta} \varphi_{t}(\eta)\right\rangle_{\varphi_{t}(\theta)}=\left\langle J_{\theta} \zeta, \eta\right\rangle_{\theta}$;

d) $\langle,\rangle_{\theta}$ and $J_{\theta}$ are $C^{\infty}$ functions of $\theta$.

We shall say that a subspace $S \subset N(\theta)$ is Lagrangian if its orthogonal complement $S^{\perp}$ is $J_{\theta} S$.

These properties can be translated into symplectic terms by defining, for each $\theta \in S M$, a 2-form $\omega_{\theta}: N(\theta) \times N(\theta) \rightarrow \mathbf{R}$ by

$$
\omega_{\theta}(\zeta, \eta)=\left\langle J_{\theta} \zeta, \eta\right\rangle_{\theta}
$$

Then $\omega_{\theta}$ is non-degenerate (by (a)) and $d \varphi_{t}$-invariant (by (c)). A subspace $S$ is now Lagrangian if and only if $\operatorname{dim} S=\operatorname{dim} N(\theta) / 2$ and $\left.\omega_{\theta}\right|_{S \times S}=0$. By property (b), $V(\theta)$ is a Lagrangian subspace for all $\theta$.

The above discussion presents the properties of $\langle,\rangle_{\theta}$ and $J_{\theta}$ that will be used here. We now give their definitions. First we define the so-called connector map $K_{\theta}: T_{\theta} S M \rightarrow T_{x} M$. Given $\zeta \in T T_{\theta} S M$, let $\theta(s)=(x(s), v(s))$ be a curve in $S M$ such that $\theta(0)=\theta$ and $\dot{\theta}(0)=$ $\zeta$. Then $K_{\theta}(\zeta)=D v / d s(0)$, i.e., $K_{\theta}(\zeta)$ is the covariant derivative at $s=0$ of the vector field $v(s)$ along the curve $x(s)$. There is a linear isomorphism $i_{\theta}: N(\theta) \rightarrow E(\theta) \oplus E(\theta)$ defined by

$$
i_{\theta}(\zeta)=\left(d_{\theta} \pi \zeta, K_{\theta} \zeta\right)
$$


The map $J_{\theta}$ is the pullback by $i_{\theta}$ of the rotation $\left(v, v^{\prime}\right) \rightarrow\left(v^{\prime},-v\right)$ on $E(\theta) \oplus E(\theta)$, and

$$
\langle\zeta, \eta\rangle_{\theta}=\left\langle d_{\theta} \pi \zeta, d_{\theta} \pi \eta\right\rangle+\left\langle K_{\theta} \zeta, K_{\theta} \eta\right\rangle .
$$

The proof of Theorem 1.1 consists of proving the following inequalities:

$$
\begin{aligned}
& \text { A) } \liminf _{T \rightarrow+\infty} \frac{1}{T} \log \int_{M \times M} n_{T}(x, y) d x d y \\
& \geq \liminf _{T \rightarrow+\infty} \frac{1}{T} \log \int_{S M} e x\left(d_{\theta} \varphi_{T}\right) d \theta . \\
& \left.A^{\prime}\right) \quad \limsup _{T \rightarrow+\infty} \frac{1}{T} \log \int_{M \times M} n_{T}(x, y) d x d y \\
& \geq \limsup _{T \rightarrow+\infty} \frac{1}{T} \log \int_{S M} \operatorname{ex}\left(d_{\theta} \varphi_{T}\right) d \theta . \\
& \text { B) } \limsup _{T \rightarrow+\infty} \frac{1}{T} \log \int_{M \times M} n_{T}(x, y) d x d y \leq h_{\text {top }}(\varphi) \text {. }
\end{aligned}
$$

These inequalities, plus Przytycki's inequality imply Theorem 1.1.

First we shall prove (A) and $\left(\mathrm{A}^{\prime}\right)$. For this purpose, and also for the proof of inequality $(\mathrm{B})$, we shall introduce, following Berger and Bott [2], a number $A(\theta, t)$ associated to each $\theta=(x, v) \in S M$ and $t>0$, defined by

$$
A(\theta, t)=\left|\operatorname{det}\left(\left.d_{\theta}\left(\pi \circ \varphi_{t}\right)\right|_{V(\theta)}\right)\right| .
$$

Berger and Bott proved ([2])

$$
\int_{0}^{T} \int_{S_{x} M} A((x, v), t) d v d t=\int_{M} n_{T}(x, y) d y .
$$

Integrating this equality over $M$ we obtain

$$
\int_{0}^{T} \int_{S M} A(\theta, t) d \theta d t=\int_{M \times M} n_{T}(x, y) d x d y .
$$

Next observe that:

$$
\begin{aligned}
\liminf _{T \rightarrow+\infty} \frac{1}{T} \log \int_{S M} & \operatorname{ex}\left(d_{\theta} \varphi_{T}\right) d \theta \\
& =\liminf _{T \rightarrow+\infty} \frac{1}{T} \log \int_{0}^{T} \int_{S M} e x\left(d_{\theta} \varphi_{t}\right) d \theta d t
\end{aligned}
$$


and

$$
\begin{aligned}
\limsup _{T \rightarrow+\infty} \frac{1}{T} \log \int_{S M} \operatorname{ex}\left(d_{\theta} \varphi_{T}\right) d \theta \\
=\limsup _{T \rightarrow+\infty} \frac{1}{T} \log \int_{0}^{T} \int_{S M} \operatorname{ex}\left(d_{\theta} \varphi_{t}\right) d \theta d t .
\end{aligned}
$$

Therefore the proof of $(\mathrm{A})$ and $\left(\mathrm{A}^{\prime}\right)$ is reduced to showing:

$$
\begin{aligned}
\liminf _{T \rightarrow+\infty} \frac{1}{T} \log & \int_{0}^{T} \int_{S M} A(\theta, t) d \theta d t \\
& \geq \liminf _{T \rightarrow+\infty} \frac{1}{T} \log \int_{0}^{T} \int_{S M} e x\left(d_{\theta} \varphi_{t}\right) d \theta d t
\end{aligned}
$$

and

$$
\begin{aligned}
\limsup _{T \rightarrow+\infty} \frac{1}{T} \log & \int_{0}^{T} \int_{S M} A(\theta, t) d \theta d t \\
& \geq \limsup _{T \rightarrow+\infty} \frac{1}{T} \log \int_{0}^{T} \int_{S M} e x\left(d_{\theta} \varphi_{t}\right) d \theta d t .
\end{aligned}
$$

Before going into the formal proof of these inequalities, let us informally describe the strategy we shall follow. Obviously the proof will rely on the analysis of the maps $d_{\theta} \varphi_{t}: N(\theta) \rightarrow N\left(\varphi_{t}(\theta)\right)$. The key idea is that the analysis becomes more accurate for our objective if we shift the initial point $\theta$ backwards to a certain point $\varphi_{-\tau_{1}}(\theta), \tau_{1}>0$, and shift the final point $\varphi_{t}(\theta)$ forward to $\varphi_{t+\tau_{2}}(\theta), \tau_{2}>0$. It will be important that these numbers $\tau_{1}$ and $\tau_{2}$ can be chosen so that they are measurable functions of the pair $(\theta, t)$, and their possible values lie in a finite set of the form $\{0,1 / m, 2 / m, \ldots, 1\}$, where $m \geq 1$ is an integer. Clearly inequalities (0.1) and (0.3) follow from

Lemma 2.1. There exists $C>0$ such that for all $T>0$,

$$
\int_{0}^{T+2} \int_{S M} A(\theta, t) d \theta d t \geq C \int_{0}^{T} \int_{S M} e x\left(d_{\theta} \varphi_{t}\right) d \theta d t
$$

To prove this lemma we first introduce a definition. For subspaces $S_{1}, S_{2}$ of $N(\theta)$ with $\operatorname{dim} S_{1}=\operatorname{dim} S_{2}=\operatorname{dim} N(\theta) / 2$, we define

$$
\alpha\left(S_{1}, S_{2}\right)=\left|\operatorname{det}\left(\left.P\right|_{S_{1}}\right)\right|
$$


where $P: N(\theta) \rightarrow S_{2}^{\perp}$ is the orthogonal projection. Clearly $\alpha$ depends continuously on the subspaces and $\alpha\left(S_{1}, S_{2}\right)=0$ if and only if $S_{1} \cap S_{2} \neq$ $\{0\}$.

The next two lemmas will be proved later.

Lemma 2.2. For each $\theta \in S M$ and $t \in \mathbf{R}$, there is a Lagrangian subspace $R_{t}(\theta) \subset N(\theta)$, which depends measurably on $t$ and $\theta$, and satisfies:

a) $\left|\operatorname{det}\left(\left.d_{\theta} \varphi_{t}\right|_{R_{t}(\theta)}\right)\right|=e x\left(d_{\theta} \varphi_{t}\right)$;

b) if $S$ is a subspace of $N(\theta)$ with $\operatorname{dim} S=\operatorname{dim} N(\theta) / 2$, then

$$
\left|\operatorname{det}\left(\left.d_{\theta} \varphi_{t}\right|_{S}\right)\right| \geq \alpha\left(S, R_{t}^{\perp}(\theta)\right) \operatorname{ex}\left(d_{\theta} \varphi_{t}\right)
$$

Lemma 2.3. There exist $\delta>0$, an integer $m \geq 1$ and measurable functions $\tau_{i}: S M \times \mathbf{R} \rightarrow\{0,1 / m, 2 / m, \ldots, 1\}, i=1,2$, such that, after abbreviating $\tau_{i}(\theta, t)$ to $\tau_{i}$ for $i=1,2$ and setting $\tau=\tau(\theta, t)=\tau_{1}+\tau_{2}$,

$$
\theta_{1}=\varphi_{-\tau_{1}}(\theta), \quad \theta_{2}=\varphi_{t+\tau_{2}}(\theta), \quad \text { and } \quad V_{i}=V\left(\theta_{i}\right) \text { for } i=1,2 \text {, }
$$

we have for all $\theta$ and $t$ :

$$
\text { a) } \alpha\left(\left(d_{\theta_{1}} \varphi_{\tau_{1}}\right) V_{1}, R_{t}^{\perp}(\theta)\right) \geq \delta
$$

and

b) $\alpha\left(\left(d_{\theta_{1}} \varphi_{t+\tau}\right) V_{1}, V_{2}\right) \geq \delta$.

From these two lemmas we shall deduce the following:

Corollary 2.4. There exists $C>0$ such that for all $t>0$ and $\theta \in S M$, the functions $\tau_{1}$ and $\tau$ given by Lemma 2.3 satisfy

$$
A\left(\varphi_{-\tau_{1}(\theta, t)}(\theta), t+\tau(\theta, t)\right) \geq C \operatorname{ex}\left(d_{\theta} \varphi_{t}\right)
$$

Proof of Corollary 2.4. Set $S=\left(d_{\theta_{1}} \varphi_{\tau_{1}}\right) V_{1}$. By Lemma 2.2 and property (a) of Lemma 2.3 we have

$$
\left|\operatorname{det}\left(\left.d_{\theta} \varphi_{t}\right|_{S}\right)\right| \geq \alpha\left(S, R_{t}^{\perp}(\theta)\right) \operatorname{ex}\left(d_{\theta} \varphi_{t}\right) \geq \delta \operatorname{ex}\left(d_{\theta} \varphi_{t}\right) .
$$

Take $C_{1}>0$ such that

$$
\left|\operatorname{det}\left(\left.d_{\zeta} \varphi_{s}\right|_{L}\right)\right| \geq C_{1}
$$


for every $\zeta \in S M, s \in[0,1]$, and every non-trivial linear subspace $L \subset N(\zeta)$. Set $\widehat{\theta}=\varphi_{t}(\theta)$ and $\widehat{S}=d_{\theta} \varphi_{t} S$. Then equations $(0.4)$ and (0.5) imply

$$
\begin{aligned}
& \left|\operatorname{det}\left(\left.d_{\theta_{1}} \varphi_{t+\tau}\right|_{V_{1}}\right)\right| \\
& \quad=\left|\operatorname{det}\left(\left.d_{\widehat{\theta}} \varphi_{\tau_{2}}\right|_{\widehat{S}}\right)\right| \cdot\left|\operatorname{det}\left(d_{\theta} \varphi_{t} \mid S\right)\right| \cdot\left|\operatorname{det}\left(\left.d_{\theta_{1}} \varphi_{\tau_{1}}\right|_{V_{1}}\right)\right| \\
& \quad \geq C_{1}^{2} \delta \operatorname{ex}\left(d_{\theta} \varphi_{t}\right) .
\end{aligned}
$$

Now set $S_{2}=\left(d_{\theta_{1}} \varphi_{t+\tau}\right) V_{1}$. By property (b) of Lemma 2.3 and the definition of $\alpha$ we have respectively

$$
\begin{gathered}
\alpha\left(S_{2}, V_{2}\right) \geq \delta, \\
\left|\operatorname{det}\left(\left.d_{\theta_{2}} \pi\right|_{S_{2}}\right)\right|>\delta,
\end{gathered}
$$

which together with inequality (0.6) implies that

$$
\begin{aligned}
A\left(\varphi_{-\tau_{1}(\theta, t)}(\theta), t+\tau(\theta, t)\right) & =\left|\operatorname{det}\left(\left.d_{\theta_{1}}\left(\pi \circ \varphi_{t+\tau}\right)\right|_{V_{1}}\right)\right| \\
& =\left|\operatorname{det}\left(\left.d_{\theta_{2}} \pi\right|_{S_{2}}\right)\right| \cdot\left|\operatorname{det}\left(\left.d_{\theta_{1}} \varphi_{t+\tau}\right|_{V_{1}}\right)\right| \\
& \geq \delta \mid \operatorname{det}\left(\left.d_{\theta_{1}} \varphi_{t+\tau}\right|_{V_{1}} \mid \geq \delta^{2} C_{1}^{2} \operatorname{ex}\left(d_{\theta} \varphi_{t}\right) .\right.
\end{aligned}
$$

Hence the proof of the corollary with $C=\delta^{2} C_{1}^{2}$ is completed.

Before proving Lemmas 2.2 and 2.3, let us see how Lemma 2.1 follows from the corollary.

Define $F: S M \times[0, T] \rightarrow S M \times[0, T+2]$ by

$$
\left.F(\theta, t)=\left(\varphi_{-\tau_{1}(\theta, t)}(\theta), t+\tau(\theta, t)\right)\right),
$$

where $\tau_{1}(\theta, t)$ and $\tau(\theta, t)$ are defined as in Lemma 2.3. Given integers $0 \leq i \leq m, 0 \leq j \leq m$, we define

$$
A(i, j)=\left\{(\theta, t) \in S M \times[0, T]: \quad \tau_{1}(\theta, t)=i / m, \quad \tau_{2}(\theta, t)=j / m\right\} .
$$

On each $A(i, j), F$ is injective, and if we set $d \mu=d \theta d t$, then $\mu(F(S))=$ $\mu(S)$ for every Borel set $S \subset A(i, j)$. Hence for any integrable function $\Phi: S M \times[0, T+2] \rightarrow \mathbf{R}$ we have

$$
\int_{F(A(i, j))} \Phi d \mu=\int_{A(i, j)}(\Phi \circ F) d \mu .
$$


Suppose now that $\Phi>0$. Then

$$
\begin{aligned}
\int_{S M \times[0, T]}(\Phi \circ F) d \mu & =\int_{\bigcup_{i, j} A(i, j)}(\Phi \circ F) d \mu=\sum_{i, j} \int_{A(i, j)}(\Phi \circ F) d \mu \\
& =\sum_{i, j} \int_{F(A(i, j))} \Phi d \mu \leq \sum_{i, j} \int_{S M \times[0, T+2]} \Phi d \mu \\
& =(m+1)^{2} \int_{S M \times[0, T+2]} \Phi d \mu .
\end{aligned}
$$

From this inequality and Corollary 2.4, it follows that

$$
\begin{aligned}
& \int_{0}^{T+2} \int_{S M} A(\theta, t) d \theta d t \\
& \quad \geq \frac{1}{(m+1)^{2}} \int_{S M \times[0, T]} A(F(\theta, t)) d \mu \\
& \quad=\frac{1}{(m+1)^{2}} \int_{0}^{T} \int_{S M} A\left(\varphi_{-\tau_{1}(\theta, t)}(\theta), t+\tau(\theta, t)\right) d \theta d t \\
& \geq \frac{C}{(m+1)^{2}} \int_{0}^{T} \int_{S M} e x\left(d_{\theta} \varphi_{t}\right) d \theta d t,
\end{aligned}
$$

thus completing the proof of Lemma 2.1.

Proof of Lemma 2.2. Consider the polar decomposition

$$
d_{\theta} \varphi_{t}=L_{t}(\theta) O_{t}(\theta)
$$

where $L_{t}(\theta): N(\theta) \rightarrow N(\theta)$ is symmetric and positive, and $O_{t}(\theta)$ : $N(\theta) \rightarrow N\left(\varphi_{t}(\theta)\right)$ is an isometry, both being $C^{\infty}$ functions of $\theta$. Since $L_{t}(\theta)=\left(\left(d_{\theta} \varphi_{t}\right)^{*}\left(d_{\theta} \varphi_{t}\right)\right)^{1 / 2}$ and $\left(d_{\theta} \varphi_{t}\right)^{*}$ is symplectic (because so is $\left.d_{\theta} \varphi_{t}\right), L_{t}(\theta)$ is symplectic and symmetric. Thus, if $\zeta$ is an eigenvector of $L_{t}(\theta)$ associated to an eigenvalue $\lambda$, then $J_{\theta} \zeta$ is an eigenvector associated to the eigenvalue $\lambda^{-1}$ because $L_{t}(\theta) J_{\theta}=J_{\theta} L_{t}(\theta)^{-1}$ (by the symmetry and the symplecticity of $\left.L_{t}(\theta)\right)$ and hence

$$
L_{t}(\theta) J_{\theta} \zeta=J_{\theta} L_{t}(\theta)^{-1} \zeta=\lambda^{-1} J_{\theta} \zeta
$$

Using this property it is possible to construct for each $t$ an orthonormal basis of $N(\theta)$ of the form $\left\{\zeta_{1}, \ldots, \zeta_{n-1}, J_{\theta} \zeta_{1}, \ldots, J_{\theta} \zeta_{n-1}\right\}$, where $\zeta_{i}$ is an eigenvector of $L_{t}(\theta)$ associated to an eigenvalue $\lambda_{i} \geq 1$. Let $R_{t}(\theta)$ be the subspace spanned by $\left\{\zeta_{1}, \ldots, \zeta_{n-1}\right\}$. Clearly $R_{t}(\theta)$ is Lagrangian and satifies property (a). 
To prove property (b), observe first that

$$
\left|\operatorname{det}\left(\left.d_{\theta} \varphi_{t}\right|_{S}\right)\right|=\left|\operatorname{det}\left(\left.L_{t}(\theta)\right|_{S}\right)\right|
$$

because $O_{t}(\theta)$ is an isometry. Notice also that $L_{t}(\theta)$ leaves $R_{t}(\theta)$ and $R_{t}^{\perp}(\theta)$ invariant, because both of these spaces are spanned by the eigenvectors of $L_{t}(\theta)$. Hence $L_{t}(\theta)$ commutes with the orthogonal projection $P: N(\theta) \rightarrow R_{t}(\theta)$, i.e., $L_{t}(\theta) \circ P=P \circ L_{t}(\theta)$. Let us suppose that $S \cap R_{t}^{\perp}(\theta)=\{0\}$, otherwise there is nothing to prove. Then $P(S)=R_{t}(\theta)$ and thus

$$
\begin{aligned}
\left|\operatorname{det}\left(\left.L_{t}(\theta)\right|_{R_{t}(\theta)}\right)\right|\left|\operatorname{det}\left(\left.P\right|_{S}\right)\right| & =\left|\operatorname{det}\left(\left.P\right|_{L_{t}(\theta)(S)}\right)\right|\left|\operatorname{det}\left(\left.L_{t}(\theta)\right|_{S}\right)\right| \\
& \leq\left|\operatorname{det}\left(\left.L_{t}(\theta)\right|_{S}\right)\right| .
\end{aligned}
$$

Hence

$$
\operatorname{ex}\left(d_{\theta} \varphi_{t}\right) \alpha\left(S, R_{t}^{\perp}(\theta)\right) \leq\left|\operatorname{det}\left(\left.d_{\theta} \varphi_{t}\right|_{S}\right)\right| .
$$

Finally we show the measurability of $R_{t}(\theta)$ as a function of $t$ and $\theta$. Let $\mathcal{F}$ denote the vector bundle over $S M$ consisting of pairs $(\theta, g)$, in which $\theta \in S M$ and $g: N(\theta) \rightarrow N(\theta)$ is a symmetric linear map. Given positive integers $p$ and $l_{i}, 1 \leq i \leq p$, let $\mathcal{F}\left(p, l_{1}, \ldots l_{p}\right)$ be the set of pairs $(\theta, g) \in \mathcal{F}$, where $g$ has $p$ eigenvalues $\lambda_{1}<\cdots<\lambda_{p}$ with multiplicities $l_{1}, \ldots, l_{p}$. Then $\mathcal{F}\left(p, l_{1}, \ldots l_{p}\right)$ is a Borel set (check it) and so is the subset $\mathcal{P}\left(p, l_{1}, \ldots, l_{p}\right)$ of $S M \times \mathbf{R}$ defined by $\left\{(\theta, t): L_{t}(\theta) \in \mathcal{F}\left(p, l_{1}, \ldots, l_{p}\right)\right\}$. Now observe that $R_{t}(\theta)$ can be chosen to be continuous on each set $\mathcal{P}\left(p, l_{1}, \ldots, l_{p}\right)$. Since these sets are Borel and there are finitely many of them, the measurability is proved.

Proof of Lemma 2.3. It suffices to prove that we can find $\delta_{1}, \delta_{2}>$ 0 , integers $m_{1}, m_{2} \geq 1$ and measurable functions $\tau_{i}: S M \times \mathbf{R} \rightarrow$ $\{0,1 / m, 2 / m, \ldots, 1\}$ such that properties (a) and (b) of Lemma 2.3 hold with $\delta$ changed to $\delta_{1}$ in (a) and to $\delta_{2}$ in (b). Then we can easily obtain Lemma 2.3 with $m=m_{1} m_{2}$ and $\delta=\min \left(\delta_{1}, \delta_{2}\right)$.

We shall prove first the existence of $\tau_{1}$ and $\tau_{2}$. The measurability will be discussed after that. We shall use the following well known property of the vertical subbundle: if $\theta \in S M$ and $S \subset N(\theta)$ is a Lagrangian subspace, then the set of values $s \in \mathbf{R}$ such that

$$
d_{\theta} \varphi_{s}(S) \cap V\left(\varphi_{s}(\theta)\right) \neq\{0\}
$$

is discrete. 
We begin by proving the existence of $\tau_{1}$. Suppose by contradiction that it does not exist. Then for every integer $m \geq 1$, there exists $\left(\theta_{m}, t_{m}\right) \in S M \times \mathbf{R}$ such that for all $s \in\{0,1 / m, 2 / m, \ldots, 1\}$ we have

$$
\alpha\left(d_{\varphi_{-s}\left(\theta_{m}\right)} \varphi_{s}\left(V\left(\varphi_{-s}\left(\theta_{m}\right)\right)\right), R_{t_{m}}^{\perp}\left(\theta_{m}\right)\right) \leq 1 / m .
$$

Since $M$ is compact, the sequence $\left(\theta_{m}, R_{t_{m}}^{\perp}\left(\theta_{m}\right)\right)$ has a subsequence $\left(\theta_{m_{k}}, R_{t_{m_{k}}}^{\perp}\left(\theta_{m_{k}}\right)\right)$ that converges to $(\theta, S)$, where $S$ is a Lagrangian subspace. From equation (0.8) and the continuity of $\alpha$ we deduce

$$
\alpha\left(d_{\varphi_{-s}(\theta)} \varphi_{s}\left(V\left(\varphi_{-s}(\theta)\right)\right), S\right)=0,
$$

for all $s \in[0,1]$. This is equivalent to

$$
d_{\varphi_{-s}(\theta)} \varphi_{s}\left(V\left(\varphi_{-s}(\theta)\right)\right) \cap S \neq\{0\},
$$

for all $s \in[0,1]$. Hence

$$
d_{\theta} \varphi_{-s}(S) \cap V\left(\varphi_{-s}(\theta)\right) \neq\{0\},
$$

for all $s \in[0,1]$. This contradicts the property of Lagrangian subspaces mentioned above.

Now we shall prove the existence of $\tau_{2}$. Suppose that it does not exist. Then there exists a sequence $\left(\theta_{m}, t_{m}\right) \in S M \times \mathbf{R}$, such that after setting $\tau_{1}(m)=\tau_{1}\left(\theta_{m}, t_{m}\right)$ and $\beta_{m}=\varphi_{-\tau_{1}(m)}\left(\theta_{m}\right)$, we have

$$
\alpha\left(d_{\beta_{m}} \varphi_{\tau_{1}(m)+s+t_{m}}\left(V\left(\beta_{m}\right)\right), V\left(\varphi_{t_{m}+s}\left(\theta_{m}\right)\right)\right) \leq 1 / m
$$

for all $s \in\{0,1 / m, 2 / m, \ldots, 1\}$. By compactness, there is a subsequence

$$
\left(\varphi_{t_{m_{k}}}\left(\theta_{m_{k}}\right), d_{\beta_{m_{k}}} \varphi_{\tau_{1}\left(m_{k}\right)+t_{m_{k}}}\left(V\left(\beta_{m_{k}}\right)\right)\right)
$$

which converges to $(\theta, S)$, where $S$ is a Lagrangian subspace. From equation (0.9) and the continuity of $\alpha$, we deduce

$$
\alpha\left(d_{\theta} \varphi_{s}(S), V\left(\varphi_{s}(\theta)\right)\right)=0
$$

for all $s \in[0,1]$. Therefore

$$
d_{\theta} \varphi_{s}(S) \cap V\left(\varphi_{s}(\theta)\right) \neq\{0\},
$$

for all $s \in[0,1]$. Since $S$ is Lagrangian, this again contradicts the above property and completes the proof of the existence of the functions $\tau_{1}$ and $\tau_{2}$. 
The measurability follows easily from observing that they can be taken locally constant on each of the subsets $\mathcal{P}\left(p, l_{1}, \ldots, l_{p}\right)$, which we defined while proving Lemma 2.2. This finishes the proof of Inequalities (A) and $\left(\mathrm{A}^{\prime}\right)$.

Now let us prove Inequality (B). We shall use a slightly strengthened version of Yomdin's theorem combined with the method employed by G. Paternain [6] in his proof that for every $x \in M$,

$$
\limsup _{T \rightarrow+\infty} \frac{1}{T} \log \int_{M} n_{T}(x, y) d y \leq h_{t o p}(\varphi) .
$$

Let $N$ be a closed manifold and $f: N \rightarrow N$ a $C^{r}$ diffeomorphism. We shall consider $N$ embedded in a Euclidean space $\mathbf{R}^{m}$ and $f$ extended to a $C^{r} \operatorname{map} f: U \rightarrow U$, where $U$ is an open neighborhood of $N$. Given a $C^{r} \operatorname{map} g: U \rightarrow \mathbf{R}^{m}$, we define

$$
\left\|d_{r} g\right\|=\sup \left\{\left\|d_{x}^{k} g\right\|: \quad x \in U, \quad 1 \leq k \leq r\right\} .
$$

Fix an integer $l \geq 1$. If $Y \subset N$, we can define the $C^{r}$-size of $Y$ as an l-dimensional set to be the infimum of $s>0$ such that there exists a $C^{r}$ map $h:[0,1]^{l} \rightarrow \mathbf{R}^{m}$ satisfying

$$
h\left([0,1]^{l}\right) \supset Y \quad \text { and } \quad\left\|d_{r} h\right\| \leq s .
$$

If no such $s$ exists, the $C^{r}$ size of $Y$ is $\infty$. If $Y \subset N$ is a submanifold, the $C^{r}$ size of $Y$ will mean the $C^{r}$ size of $Y$ as a $\operatorname{dim} Y$-dimensional set. These definitions are taken from Gromov [4]. We can assume that $\operatorname{dist}(f(U), \partial U) \geq 1 / \sqrt{l}$ (as required by Gromov in [4]) by rescaling the embedding and thus increasing $\operatorname{dist}(f(U), \partial U)$.

The next result is the slight improvement of Yomdin's theorem that we shall need. For simplicity, we shall state it and prove it for the case of diffeomorphisms; however we shall use it for flows.

For the function $f$ considered above, we define

$$
K=K(f)=1+\limsup _{k \rightarrow+\infty} \frac{1}{k} \log \left\|d f^{k}\right\| .
$$

Theorem 2.5. Let $f: N \rightarrow N$ and $K=K(f)$ be as above. For any $S, \varepsilon>0$ and any integers $r, l \geq 1$, there exist $C>0$ and an integer $n_{0} \geq 1$ such that, for every l-dimensional submanifold $Y \subset N$ with $C^{r}$ size $\leq S$, we have

$$
\operatorname{Vol}\left(f^{n}(Y)\right) \leq C \exp \left\{\left(h_{\text {top }}\left(\left.f\right|_{N}\right)+\varepsilon+\frac{l K}{r}\right) n\right\}
$$

for all $n \geq n_{0}$. 
Proof. As observed by Gromov [4, 3.2 p.231], every set of $C^{r}$-size $\leq S$ can be divided into $j^{l}$ subsets of $C^{r}$-size $\leq S / j$ for all $j=1,2, \ldots$. Thus it suffices to prove the theorem in the case where $Y$ has $C^{r}$-size $\leq 1$. We shall need the following lemma proved by Gromov $[4,3.6$ p.233].

Lemma 2.6. There exists $C=C(l, m, r)$ independent of $f$, such that if $Y_{0} \subset N$ is a $C^{r}$ l-dimensional submanifold of $C^{r}$-size $\leq 1$ and $Q_{\beta}, \beta=1, \ldots, i$, are unit cubes contained in space $\mathbf{R}^{m}$ in which we embedded $N$, then

$$
\operatorname{Vol}\left(f^{i}\left(Y_{0} \cap\left(\bigcap_{b=1}^{i} f^{-\beta}\left(Q_{\beta}\right)\right)\right)\right) \leq\left(C\left\|d_{r} f\right\|^{l / r}+1\right)^{i} .
$$

Given a continuous map $g: X \rightarrow X$ of a compact metric space, denote by $n(\delta, i, g)$ the minimal cardinality of a $(\delta, i, g)$-spanning set.

Assume that the points $x_{s}, s=1, \ldots, n\left(1 / 2, i,\left.f\right|_{N}\right)$, form a $\left(1 / 2, i,\left.f\right|_{N}\right)$-spanning set. Then the manifold $N$ can be covered by sets of the form

$$
A_{s} \stackrel{\text { def }}{=} \bigcap_{\beta=1}^{i} f^{-\beta}\left(Q_{\beta}\right), \quad 1 \leq s \leq n\left(1 / 2, i,\left.f\right|_{N}\right),
$$

where $Q_{\beta}$ is the unit cube centered at $f^{\beta}\left(x_{s}\right)$. If $Y_{0}$ is as in Lemma 2.6, we have

$$
\begin{aligned}
\operatorname{Vol}\left(f^{i}\left(Y_{0}\right)\right) & \leq \sum_{s} \operatorname{Vol}\left(f^{i}\left(Y_{0} \cap A_{s}\right)\right) \\
& \leq n\left(1 / 2, i,\left.f\right|_{N}\right)\left(C\left\|d_{r} f\right\|^{l / r}+1\right)^{i}
\end{aligned}
$$

Now define $f_{j}: j U \rightarrow j U$, for $j \geq 1$, by $f_{j}(x)=j f\left(j^{-1} x\right)$. Then $f_{j}(j N)=j N$. Let $Y_{0}$ be a submanifold of $C^{r}$-size $\leq 1$. Observe that $j Y_{0}$ can be covered by $j^{l}$-sets, $Y_{0}^{k}, k=1, \ldots, j^{l}$, with $C^{r}$-size $\leq 1$ [4]. Therefore we can apply equation $(0.11)$ to each $Y_{0}^{k}$, obtaining

$$
\begin{aligned}
\operatorname{Vol}\left(f_{j}^{i}\left(j Y_{0}\right)\right) & \leq \sum_{k} \operatorname{Vol}\left(f_{j}^{i}\left(Y_{0}^{k}\right)\right) \\
& \leq j^{l} n\left(1 / 2, i,\left.f_{j}\right|_{j N}\right)\left(C\left\|d_{r} f_{j}\right\|^{l / r}+1\right)^{i} .
\end{aligned}
$$

But

$$
n\left(1 / 2, i,\left.f_{j}\right|_{j N}\right)=n\left(\frac{1}{2 j}, i,\left.f\right|_{N}\right)
$$


By the definition of topological entropy, given $\delta>0$, there exist $i_{0} \geq 1$ and $j_{0} \geq 1$ such that

$$
n\left(\frac{1}{2 j}, i,\left.f\right|_{N}\right) \leq \exp \left\{\left(h_{\text {top }}\left(\left.f\right|_{N}\right)+\delta\right) i\right\}
$$

for all $i \geq i_{0}, j \geq j_{0}$. Then for $i \geq i_{0}, j \geq j_{0}$, inequality (0.12) implies

$$
\begin{aligned}
\operatorname{Vol}\left(f^{i}\left(Y_{0}\right)\right) & =\operatorname{Vol}\left(j^{-1} f_{j}^{i}\left(j Y_{0}\right)\right)=j^{-l} \operatorname{Vol}\left(f_{j}^{i}\left(j Y_{0}\right)\right) \\
& \leq n\left(\frac{1}{2 j}, i,\left.f\right|_{N}\right)\left(C\left\|d_{r} f_{j}\right\|^{l / r}+1\right)^{i} \\
& \leq\left(\exp \left\{\left(h_{\text {top }}\left(\left.f\right|_{N}\right)+\delta\right) i\right\}\right) \cdot\left(C\left\|d_{r} f_{j}\right\|^{l / r}+1\right)^{i} .
\end{aligned}
$$

The definition of $K$ in equation (0.10) allows us to choose $k$ so that

$$
\frac{1}{k} \log \left(C\left(2|| d f^{k}||\right)^{l / r}+1\right) \leq \frac{l K}{r} .
$$

Observe that $\left\|d^{s} f_{j}\right\|=j^{1-s}\left\|d^{s} f\right\|$ for $s=1,2, \ldots$. We see from this that we can choose $j \geq j_{0}$ such that

$$
\left\|d_{r} f_{j}^{k}\right\| \leq 2\left\|d f^{k}\right\|
$$

If $i \geq i_{0}$, then inequality $(0.13)$ applied to $f^{k}$ with $\delta=k \varepsilon$ gives

$$
\begin{aligned}
\operatorname{Vol}\left(f^{k i}\left(Y_{0}\right)\right) & \leq\left(\exp \left\{\left(h_{\text {top }}\left(\left.f^{k}\right|_{N}\right)+k \varepsilon\right) i\right\}\right) \cdot\left(C\left\|d_{r} f_{j}^{k}\right\|^{l / r}+1\right)^{i} \\
& \leq \exp \left\{\left(k h_{\text {top }}\left(\left.f\right|_{N}\right)+k \varepsilon+\log \left(C\left(2\left\|d f^{k}\right\|\right)^{l / r}+1\right)\right) i\right\} \\
& \leq \exp \left\{\left(h_{\text {top }}\left(\left.f\right|_{N}\right)+\varepsilon+\frac{l K}{r}\right) k i\right\} .
\end{aligned}
$$

If $n \geq k i_{0}$, we can choose $i \geq i_{0}$ such that $0 \leq n-k i<k$. For $n \geq k i_{0}$, we have

$$
\begin{aligned}
\operatorname{Vol}\left(\left(f^{n}\left(Y_{0}\right)\right)\right. & =\operatorname{Vol}\left(f^{n-k i}\left(f^{k i}\left(Y_{0}\right)\right)\right) \\
& \leq\|d f\|^{l(n-k i)} \exp \left\{\left(h_{t o p}\left(\left.f\right|_{N}\right)+\varepsilon+\frac{l K}{r}\right) k i\right\} \\
& \leq C \exp \left\{\left(h_{\text {top }}\left(\left.f\right|_{N}\right)+\varepsilon+\frac{l K}{r}\right) n\right\}
\end{aligned}
$$

where $C=\|d f\|^{l k}$. This completes the proof of the theorem. 
Now let us complete the proof of Inequality (B). Observe that

$$
\int_{S_{x} M} A((x, v), t) d v \leq \operatorname{Vol}\left(\varphi_{t}\left(S_{x} M\right)\right)
$$

By the theorem above, given $\varepsilon>0$ and an integer $r \geq 1$, there exist $t_{0} \geq 0$ and $C_{r}>0$ such that

$$
\operatorname{Vol}\left(\varphi_{t}\left(S_{x} M\right)\right) \leq C_{r} \exp \left\{\left(h_{t o p}(\varphi)+\varepsilon+\frac{(n-1) K}{r}\right) t\right\},
$$

for all $t \geq t_{0}$ and all $x \in M$, since it is easy to see (using the compactness of $M$ ) that there exists $S>0$ such that the $(n-1)$-dimensional manifold $S_{x} M$ has $C^{r}$-size $\leq S$ for all $x \in M$. Hence there is a constant $C_{r}^{\prime}$ such that, for all large enough $T$, we have

$$
\begin{aligned}
\int_{M \times M} n_{T}(x, y) d x d y & =\int_{0}^{T} \int_{M} \int_{S_{x} M} A(x, v, t) d v d x d t \\
& \leq C_{r}^{\prime} \exp \left\{\left(h_{\text {top }}(\varphi)+\varepsilon+\frac{(n-1) K}{r}\right) T\right\} .
\end{aligned}
$$

Therefore

$$
\limsup _{T \rightarrow+\infty} \frac{1}{T} \log \int_{M \times M} n_{T}(x, y) d x d y \leq h_{t o p}(\varphi)+\varepsilon+\frac{(n-1) K}{r},
$$

concluding the proof of Inequality (B), since the above inequality holds for all $\varepsilon>0$ and all $r \geq 1$.

\section{Proof of Theorem 1.4}

We shall use here the following lemma.

Lemma 3.1. There exist a constant $K>0$, an integer $m \geq 1$ and a measurable function $\tau: S M \times \mathbf{R}^{+} \rightarrow\{0,1 / m, 2 / m, \ldots, 1\}$ such that, after setting

$$
G_{T}(\theta)=\varphi_{-\tau(\theta, T)} \theta
$$

we have

$$
\left|\operatorname{det}\left(\left.d_{G_{T}(\theta)} \varphi_{T}\right|_{V\left(G_{T}(\theta)\right)}\right)\right| \geq K \operatorname{ex}\left(d_{\theta} \varphi_{T}\right)
$$

for any $T>0$. 
We shall not prove this lemma because it is obtained by the same methods as the lemmas of Section 2.

Now, given $T>0$, we prove as in Section 2, that there exists $K_{1}>0$ (independent of $T$ ) such that

$$
\int_{S M}\left(f \circ G_{T}\right) d \theta \leq K_{1} \int_{S M} f d \theta
$$

for every integrable function $f: S M \rightarrow(0,+\infty)$. Hence

$$
\begin{aligned}
\int_{S M} \operatorname{ex}\left(d_{\theta} \varphi_{T}\right) d \theta & \leq K^{-1} \int_{S M}\left|\operatorname{det}\left(\left.d_{G_{T}(\theta)} \varphi_{T}\right|_{V\left(G_{T}(\theta)\right)}\right)\right| d \theta \\
& \leq K_{1} K^{-1} \int_{S M}\left|\operatorname{det}\left(\left.d_{\theta} \varphi_{T}\right|_{V(\theta)}\right)\right| d \theta
\end{aligned}
$$

and in consequence of Theorem 1.1,

$$
\begin{aligned}
h_{\text {top }}(\varphi) & =\lim _{T \rightarrow+\infty} \frac{1}{T} \log \int_{S M} \operatorname{ex}\left(d_{\theta} \varphi_{T}\right) d \theta \\
& \leq \liminf _{T \rightarrow+\infty} \frac{1}{T} \log \int_{S M}\left|\operatorname{det}\left(\left.d_{\theta} \varphi_{T}\right|_{V(\theta)}\right)\right| d \theta .
\end{aligned}
$$

Since $\operatorname{ex}\left(d_{\theta} \varphi_{T}\right) \geq\left|\operatorname{det}\left(\left.d_{\theta} \varphi_{T}\right|_{V(\theta)}\right)\right|$, by definition of $\operatorname{ex}($.$) , we also have:$

$$
\begin{aligned}
\limsup _{T \rightarrow+\infty} \frac{1}{T} \log \int_{S M}\left|\operatorname{det}\left(\left.d_{\theta} \varphi_{T}\right|_{V(\theta)}\right)\right| d \theta & \leq \lim _{T \rightarrow+\infty} \frac{1}{T} \log \int_{S M} \operatorname{ex}\left(d_{\theta} \varphi_{T}\right) d \theta \\
& =h_{\text {top }}(\varphi) .
\end{aligned}
$$

\section{Appendix: An upper bound for the growth rate of}

$$
n_{T}(x, y) \text {. }
$$

Here we shall prove the following property:

Proposition 4.1. For every $x \in M$,

$$
\limsup _{T \rightarrow+\infty} \frac{1}{T} \log n_{T}(x, y) \leq h_{\text {top }}(\varphi)
$$

for a.e. $x \in M$.

This is an immediate corollary of the following inequality due to G. Paternain [6]: 
Proposition 4.2. For every $x \in M$, we have

$$
\limsup _{T \rightarrow+\infty} \frac{1}{T} \log \int_{M} n_{T}(x, y) d y \leq h_{t o p}(\varphi),
$$

and the following application of the Borel-Cantelli Lemma:

Lemma 4.3. Let $(X, \mathcal{A}, \mu)$ be a probability space, and $f_{n}: X \rightarrow$ $(0,+\infty)$ a sequence of integrable functions. Then

$$
\limsup _{n \rightarrow \infty} \frac{1}{n} \log f_{n}(x) \leq \limsup _{n \rightarrow \infty} \frac{1}{n} \log \int_{X} f_{n} d \mu,
$$

for $\mu$-a.e. $x \in X$.

Proof. Set

$$
\sigma=\limsup _{n \rightarrow \infty} \frac{1}{n} \log \int_{X} f_{n} d \mu
$$

Define $S(n, \epsilon)=\left\{x: f_{n}(x) \geq \exp (\sigma+\epsilon) n\right\}$. Then

$$
\begin{aligned}
\sigma & =\limsup _{n \rightarrow \infty} \frac{1}{n} \log \int_{X} f_{n} d \mu \geq \limsup _{n \rightarrow \infty} \frac{1}{n} \log \int_{S(n, \epsilon)} f_{n} d \mu \\
& \geq \limsup _{n \rightarrow \infty} \frac{1}{n} \log [\mu(S(n, \epsilon)) \exp (\sigma+\epsilon) n] \\
& =\sigma+\epsilon+\limsup _{n \rightarrow \infty} \frac{1}{n} \log \mu(S(n, \epsilon)) .
\end{aligned}
$$

Hence

$$
\limsup _{n \rightarrow \infty} \frac{1}{n} \log \mu(S(n, \epsilon)) \leq-\epsilon
$$

which implies

$$
\sum_{n} \mu(S(n, \epsilon))<+\infty .
$$

By the Borel-Cantelli Lemma, for a.e. $x$, there exists $m(x)$ such that $x \notin S(n, \epsilon)$ for all $n \geq m(x)$. This means that $f_{n}(x) \leq \exp (\sigma+\epsilon) n$ for all $n \geq m(x)$ and then

$$
\limsup _{n \rightarrow \infty} \frac{1}{n} \log f_{n}(x) \leq \sigma+\epsilon .
$$

Since this holds for every $\epsilon>0$, the lemma is proved. 


\section{References}

[1] W. Ballmann, M. Brin \& K.Burns, On surfaces without conjugate points, J. Differential Geom. 25 (1987) 249-273.

[2] M. Berger \& R. Bott, Sur les variétés à courbure strictement positive, Topology 1 (1962) 302-311.

[3] A. Freire \& R. Mañé, On the entropy of the geodesic flow in manifolds without conjugate points, Invent. Math. 69 (1982) 375-392.

[4] M. Gromov, Entropy, homology and semialgebraic Geometry, Séminaire Bourbaki, 38éme année, 1985-86, Exp. 663, 225-240.

[5] A. Manning, Topological entropy for geodesic flows, Ann. of Math. 110 (1979) $567-573$.

[6] G. P. Paternain, On the topology of manifolds with completely integrable geodesic flows, Ergodic Theory Dynamical Systems 12 (1992) 109-121.

[7] _ M. Paternain, Topological entropy versus geodesic entropy, Internat. J. Math. 2 (1994) 213-218.

[8] F. Przytycki, An upper estimation for topological entropy of diffeomorphisms, Invent. Math. 59 (1980) 205-213.

[9] Y. Yomdin, Volume growth and entropy, Israel J. Math. 57 (1987) 287-300.

INST. MAT. PURA APL. RIO DE JANEIRO, BRAZIL 